University of Nebraska - Lincoln

DigitalCommons@University of Nebraska - Lincoln

\title{
On-line Classification of US Select Beef Carcasses for Longissimus Tenderness Using Visible and Near-Infrared Reflectance Spectroscopy
}

\author{
S. D. Shackelford \\ USDA-ARS, shackelford@email.marc.usda.gov
}

T. L. Wheeler

USDA-ARS, tommy.wheeler@ars.usda.gov

M. Koohmaraie

USDA-ARS

Follow this and additional works at: https://digitalcommons.unl.edu/hruskareports

Shackelford, S. D.; Wheeler, T. L.; and Koohmaraie, M., "On-line Classification of US Select Beef Carcasses for Longissimus Tenderness Using Visible and Near-Infrared Reflectance Spectroscopy" (2005). Roman L. Hruska U.S. Meat Animal Research Center. 252.

https://digitalcommons.unl.edu/hruskareports/252

This Article is brought to you for free and open access by the U.S. Department of Agriculture: Agricultural Research Service, Lincoln, Nebraska at DigitalCommons@University of Nebraska - Lincoln. It has been accepted for inclusion in Roman L. Hruska U.S. Meat Animal Research Center by an authorized administrator of DigitalCommons@University of Nebraska - Lincoln. 


\title{
On-line classification of US Select beef carcasses for longissimus tenderness using visible and near-infrared reflectance spectroscopy is
}

\author{
S.D. Shackelford *, T.L. Wheeler, M. Koohmaraie \\ Roman L. Hruska US Meat Animal Research Center, USDA, ARS, Clay Center, NE 68933-0166, USA
}

Received 6 January 2004; received in revised form 24 August 2004; accepted 24 August 2004

\begin{abstract}
The current experiment was conducted to evaluate the on-line application of visible and near-infrared spectroscopy (VISNIR) to US Select carcasses during commercial beef carcass grading procedures to predict tenderness of longissimus steaks after 14 days of refrigerated storage. A regression model was calibrated using 146 carcasses and tested against an additional 146 carcasses. Carcasses were segregated into VISNIR-based tenderness classes based on whether their VISNIR-predicted slice shear force value was less than (tender) or greater than (tough) the median predicted slice shear force value. Carcasses classified as tender by VISNIR had a lower mean SSF value, were less likely to have slice shear force values greater than $245 \mathrm{~N}$, had higher trained sensory panel tenderness ratings, and were less likely to have trained sensory panel tenderness ratings below slightly tender than were carcasses classified as tough $(P<0.001)$. This technology might be useful for identification of US Select carcasses that excel in longissimus tenderness.
\end{abstract}

Published by Elsevier Ltd.

Keywords: Beef; Near-infrared spectroscopy; Tenderness

\section{Introduction}

US Select cuts are currently marketed at a discount relative to US Choice cuts despite the fact that many cuts from US Select carcasses are very tender. Consumers have shown a strong willingness to pay a premium for "Tender Select" cuts that combine superior tenderness with the leanness of Select (Shackelford et al.,

\footnotetext{
On-line classification of US Select beef carcasses for longissimus tenderness using visible and near-infrared reflectance spectroscopy Online classification of US Select beef carcasses for longissimus tenderness using visible and near-infrared reflectance spectroscopy.

${ }^{*}$ Corresponding author. Tel.: +1 402762 4223; fax: +1 402762 4149.

E-mail address: shackelford@email.marc.usda.gov

(S.D.
}

2001). Therefore, meat retailers have expressed strong interest in marketing a "Tender Select" product line. However, beef packing companies have not been able to meet the needs of the retailers because non-invasive methods to predict tenderness, such as Beefcam (Belk et al., 2000) and colorimeter (Wulf \& Page, 2000), have been ineffective for classifying Select carcasses (Wheeler et al., 2002). Thus, there is a need to develop a non-invasive method to accurately identify US Select carcasses that excel in meat tenderness. Several studies have shown that near-infrared reflectance spectroscopy can be used to predict beef tenderness (Byrne, Downey, Troy, \& Buckley, 1998; Hildrum, Nilsen, Mielnik, \& Naes, 1994; Hildrum et al., 1995; Mitsumoto, Maeda, Mitsuhashi, \& Ozawa, 1991; Naes \& Hildrum, 1997; Park, Chen, Hruschka, Shackelford, \& Koohmaraie, 1998). However, the procedures used in those studies 
were either destructive in that they required excision of a muscle sample for spectroscopy (Hildrum et al., 1994, 1995; Naes \& Hildrum, 1997; Park et al., 1998) or they were limited to sampling a very small area $\left(4 \mathrm{~cm}^{2}\right)$ and thus, would be highly subject to error induced by nonrepresentative sampling of the target muscle (Byrne et al., 1998; Mitsumoto et al., 1991). We (Shackelford, Wheeler, \& Koohmaraie, 2004) developed a highly repeatable method for on-line spectroscopic evaluation of longissimus quality traits of ribbed beef carcasses using a high-intensity reflectance probe that allowed for sampling of a representative portion of the exposed longissimus cross-section of ribbed beef carcasses. Therefore, the current experiment was conducted to evaluate the on-line application of visible and near-infrared spectroscopy (VISNIR) to US Select carcasses during commercial beef carcass grading procedures to predict tenderness of longissimus steaks after 14 days of refrigerated storage.

\section{Materials and methods}

Spectroscopy was conducted on-line at two largescale commercial fed-beef processing facilities. At $24 \mathrm{~h}$ postmortem, carcasses were ribbed conventionally between the 12th and 13th ribs for determination of USDA beef quality and yield grades and spectroscopy was conducted on the longissimus cross-section. Spectroscopy was conducted on the beef grading bloom chain approximately 2 min after the carcasses were ribbed. Spectroscopy only was conducted on carcasses that were likely to be graded USDA Select and USDA yield grade 1,2 , or 3 . After grading, any carcasses that did not meet these specifications were excluded from the experiment. The carcasses that were included in the experiment had a slight amount of marbling in the longissimus which suggests that the longissimus intramuscular fat content was approximately $3.4 \%$ (Savell, Cross, \& Smith, 1986).

Spectroscopy was conducted using the optimal protocol developed by Shackelford et al. (2004). Spectroscopy was conducted using a Model A108310 LabSpec Pro portable spectrophotometer (ASD; Analytical Spectral Devices, Inc., Boulder, CO) which was equipped to collect spectra from 350 to $2500 \mathrm{~nm}$. Spectra were collected via a ASD Model 135090 2-m long fiber optic jumper cable attached to a ASD Model A122000 high-intensity reflectance probe, that served as an external light source ( $2900 \mathrm{~K}$ color temperature quartz halogen light) to illuminate the object of interest. The field of view was restricted to $50 \mathrm{~mm}$ in diameter using a modified ASD Model A122040 field of view limiter and plate. This field of view size was such that a large area of the cross-section of the longissimus was sampled.

\subsection{Slice shear force}

Following spectroscopy and grading, carcasses were fabricated and the NAMP 180 strip loin (NAMP, 1997) was obtained from the left side of each carcass $(n=292)$. Strip loins were obtained from 143 and 149 carcasses at the two respective processing facilities. Strip loins were transported to the MARC abattoir and aged $\left(1{ }^{\circ} \mathrm{C}\right)$ until $14 \mathrm{~d}$ postmortem. At $14 \mathrm{~d}$ postmortem, a 2.54-cm thick longissimus steak was removed from the anterior end of each strip loin, cooked using a belt grill as described by Wheeler, Shackelford, and Koohmaraie (1998), and slice shear force (SSF) was measured as described by Shackelford, Wheeler, and Koohmaraie (1999). The remainder of each strip loin was frozen $\left(-20^{\circ} \mathrm{C}\right)$. Subsequently, two steaks $(2.54 \mathrm{~cm}$ thick $)$ were obtained from the anterior end of each frozen strip loin for trained descriptive attribute sensory panel evaluation.

Table 1

Simple statistics of slice shear force and trained sensory panel traits for the calibration and prediction data sets

\begin{tabular}{|c|c|c|c|c|c|c|c|}
\hline Trait & $N$ & Mean & SD & Minimum & Maximum & $\%>245 \mathrm{~N}$ & $\%<5^{\mathrm{b}}$ \\
\hline \multicolumn{8}{|l|}{ Calibration data set } \\
\hline Slice shear force $(\mathrm{N})$ & 146 & 186.4 & 67.7 & 92.2 & 455.2 & 17.8 & - \\
\hline Tenderness $^{\mathrm{a}}$ & 146 & 5.3 & 0.8 & 2.4 & 6.6 & - & 28.8 \\
\hline Juiciness $^{\mathrm{a}}$ & 146 & 5.4 & 0.3 & 4.7 & 6.1 & - & - \\
\hline Beef flavor intensity ${ }^{a}$ & 146 & 5.1 & 0.3 & 4.5 & 5.8 & - & - \\
\hline \multicolumn{8}{|l|}{ Prediction data set } \\
\hline Slice shear force $(\mathrm{N})$ & 146 & 186.4 & 66.7 & 91.2 & 437.6 & 17.8 & - \\
\hline Tenderness $^{\mathrm{a}}$ & 146 & 5.4 & 0.8 & 2.3 & 7.0 & - & 23.3 \\
\hline Juiciness $^{\text {a }}$ & 146 & 5.4 & 0.3 & 4.6 & 6.1 & - & - \\
\hline Beef flavor intensity ${ }^{a}$ & 146 & 5.1 & 0.3 & 4.2 & 5.6 & - & - \\
\hline
\end{tabular}

${ }^{\mathrm{a}}$ Tenderness, juiciness, and beef flavor intensity rated on 8-point scales $(1=$ extremely tough, dry, and bland; $8=$ extremely tender, juicy, and intense).

b 5 = slightly tender. 


\subsection{Trained sensory panel}

Frozen steaks were thawed $\left(24 \mathrm{~h}\right.$ at $\left.5{ }^{\circ} \mathrm{C}\right)$ and cooked using a belt grill as described by Wheeler et al. (1998). Immediately after post-cooking temperature rise was completed, steaks were sliced and served. Each panelist received three random cubes $(1.3 \mathrm{~cm} \times 1.3 \mathrm{~cm} \times$ cooked steak thickness) from each sample. Sensory panelists scored steaks for tenderness on an eight-point scale $(1=$ extremely tough and $8=$ extremely tender $)$. The eight-member sensory panel was selected and trained according to Cross, Moen, and Stanfield (1978) and was highly experienced. With the protocol used in this experiment, the eight-member sensory panel has been reported (Wheeler et al., 1998) to measure tenderness with a high (0.87) level of repeatability.
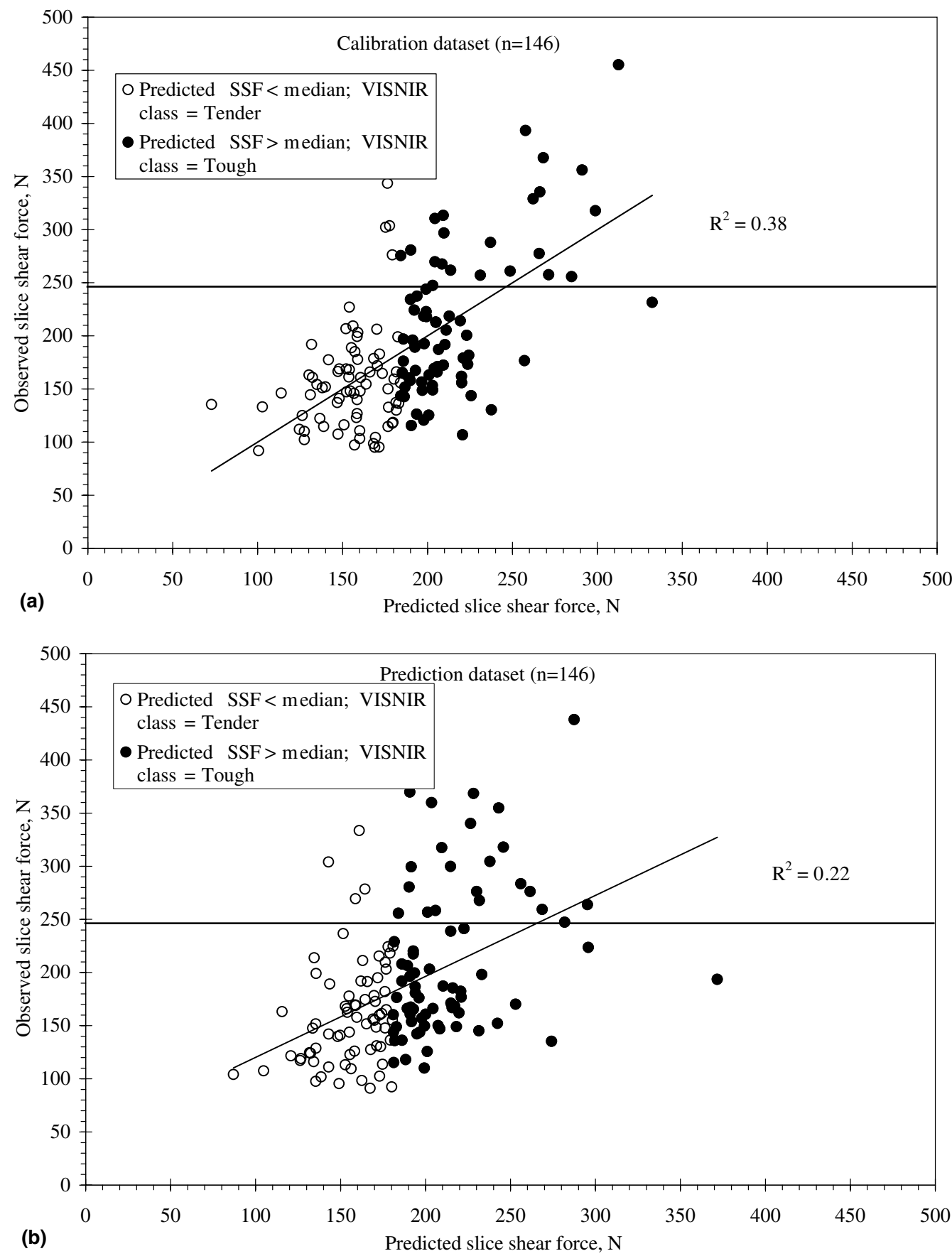

Fig. 1. Scatter plots for predicted vs observed slice shear force for the calibration (a) and prediction (b) data sets. The regression model was developed using the calibration data set and tested on the prediction data set. Open and closed circles correspond to the carcasses represented by white and black bars, respectively, in Fig. 2. 


\subsection{Statistical analysis}

To facilitate computations and to reduce spectral noise, spectra were reduced by averaging groups of nine consecutive wavelengths using Unscrambler ${ }^{\circledR}$ (Version 7.5; Camo, Inc., Corvallis, OR).

Carcasses were blocked by plant and observed slice shear force and one-half of the carcasses were assigned to a calibration data set, which was used to develop regression equations, and one-half of the carcasses were assigned to a prediction data set, which was used to validate the regression equations (Neter, Wasserman, \& Kutner, 1989).

Forwards stepwise regression (SAS Inst., Inc., Cary, NC) was used to construct 1 to 20-variable regression equation for the prediction of SSF. The 10-variable regression equation was tested against the prediction data set.

The equations were validated as follows: carcasses were classified as "Tender" if their VISNIR-predicted slice shear force value was less than the median predicted slice shear force value. Carcasses were classified as "Tough" if their VISNIR-predicted slice shear force value was greater than the median predicted slice shear force value. The median predicted slice shear force value was 184 and $182 \mathrm{~N}$ for the calibration and prediction data sets, respectively. Analysis of variance was used to determine the effect of VISNIR classification on slice shear force and trained sensory panel traits. Analysis of variance was conducted using the GLM procedure of SAS. The frequency of carcasses with slice shear force values $>245 \mathrm{~N}$ and the frequency of carcasses with trained sensory panel tenderness ratings $<5$ was calculated for each VISNIR class. Differences in these frequencies among VISNIR classes was compared using the DIFFER program of PEPI (Version 2; USD, Inc., Stone Mountain, GA).

\section{Results and discussion}

Simple statistics of the calibration and prediction data sets are presented in Table 1. The blocking method used to assign the carcasses to the calibration and prediction data sets insured that the data sets had similar simple statistics. There was ample variation in tenderness in the data sets for development and validation of procedures for prediction of tenderness.

The most variation in SSF that could be accounted for by the amount of light reflected at any single wavelength was $9.6 \%$. A 10 -variable regression equation accounted for $38 \%$ of the variation in SSF in the calibration data set (Fig. 1). When that equation was applied to the prediction data set, predicted-SSF accounted for $22 \%$ of the variation in observed SSF (Fig. 1). Although this level of accuracy would not be great enough to serve as the basis for rewarding or penalizing cattle producers for differences in raw material quality, it appears that this technology could allow the beef industry to non-invasively identify US Select carcasses whose longissimus is more consistently tender than is typical of US Select.

Although the pool of independent variables included reflectance values spanning the range from 449 to 2500 $\mathrm{nm}$, only reflectance values associated with wavelengths between 552 and $930 \mathrm{~nm}$ were included in the 10-variable equation. That is, all of the information used in the prediction of tenderness was derived from one of the spectrometer's three detectors. Thus, it appears that this level of prediction accuracy could be achieved using a much less complex instrument.

A potential use of this technology would be for identification of carcasses that excel in both leanness and tenderness for a branded product such as the Tender Select product described by Shackelford et al. (2001). There is a segment of consumers that are willing to

Table 2

Effect of VISNIR classification on slice shear force and trained sensory panel traits for the calibration and prediction data sets

\begin{tabular}{|c|c|c|c|c|c|c|}
\hline VISNIR classification $^{a}$ & $\begin{array}{l}\text { Slice shear } \\
\text { force }(\mathrm{N})\end{array}$ & $\begin{array}{l}\text { Percentage of slice shear } \\
\text { force values }>245 \mathrm{~N}\end{array}$ & $\begin{array}{l}\text { Tenderness } \\
\text { rating }{ }^{\mathrm{b}}\end{array}$ & $\begin{array}{l}\text { Percentage of tenderness } \\
\text { ratings }<5^{c}\end{array}$ & $\begin{array}{l}\text { Juiciness } \\
\text { rating }^{\mathrm{b}}\end{array}$ & $\begin{array}{l}\text { Beef flavor } \\
\text { intensity rating }\end{array}$ \\
\hline \multicolumn{7}{|l|}{ Calibration data set } \\
\hline Tender & 157 & 5.5 & 5.6 & 17.8 & 5.5 & 5.2 \\
\hline Tough & 216 & 30.1 & 5.0 & 39.7 & 5.4 & 5.1 \\
\hline SEM & 7.2 & 4.3 & 0.1 & 5.2 & 0.03 & 0.03 \\
\hline$P$ value & $<0.001$ & $<0.001$ & $<0.001$ & $<0.01$ & 0.34 & 0.11 \\
\hline \multicolumn{7}{|l|}{ Prediction data set } \\
\hline Tender & 160 & 5.5 & 5.6 & 11.0 & 5.5 & 5.1 \\
\hline Tough & 212 & 30.1 & 5.1 & 35.6 & 5.4 & 5.1 \\
\hline SEM & 7.1 & 4.3 & 0.1 & 4.8 & 0.03 & 0.03 \\
\hline$P$ value & $<0.001$ & $<0.001$ & $<0.001$ & $<0.001$ & 0.20 & 0.99 \\
\hline
\end{tabular}

" Carcasses were classified as "Tender" if their VISNIR-predicted slice shear force value was less than the median predicted slice shear force value. Carcasses were classified as "Tough" if their VISNIR-predicted slice shear force value was greater than the median predicted slice shear force value. The median predicted slice shear force value was 184 and $182 \mathrm{~N}$ for the calibration and prediction data sets, respectively.

b Tenderness, juiciness, and beef flavor intensity rated on 8-point scales $(1=$ extremely tough, dry, and bland; $8=$ extremely tender, juicy, and intense).

c $5=$ slightly tender. 
pay a premium for a product that is both lean and tender. A processing company could use this technology to select carcasses with low predicted SSF values and increase the likelihood of achieving a high level of consumer satisfaction. For example, if the processor that produced the carcasses used in this experiment had a premium product line that used $50 \%$ of its US Select carcasses, the processor could have assigned the carcasses with predicted SSF values less than the median to the premium line and reduced the frequency of longissimus SSF values greater than $245 \mathrm{~N}$ from $17.8 \%$ (Table 1) to $5.5 \%$ (Table 2) for the premium line.

When carcasses in the calibration data set were segregated into VISNIR-based tenderness classes based on whether their VISNIR-predicted slice shear force value was less than (tender) or greater than (tough) the median predicted slice shear force value, it was determined that the tender class had a lower mean SSF value, a lower
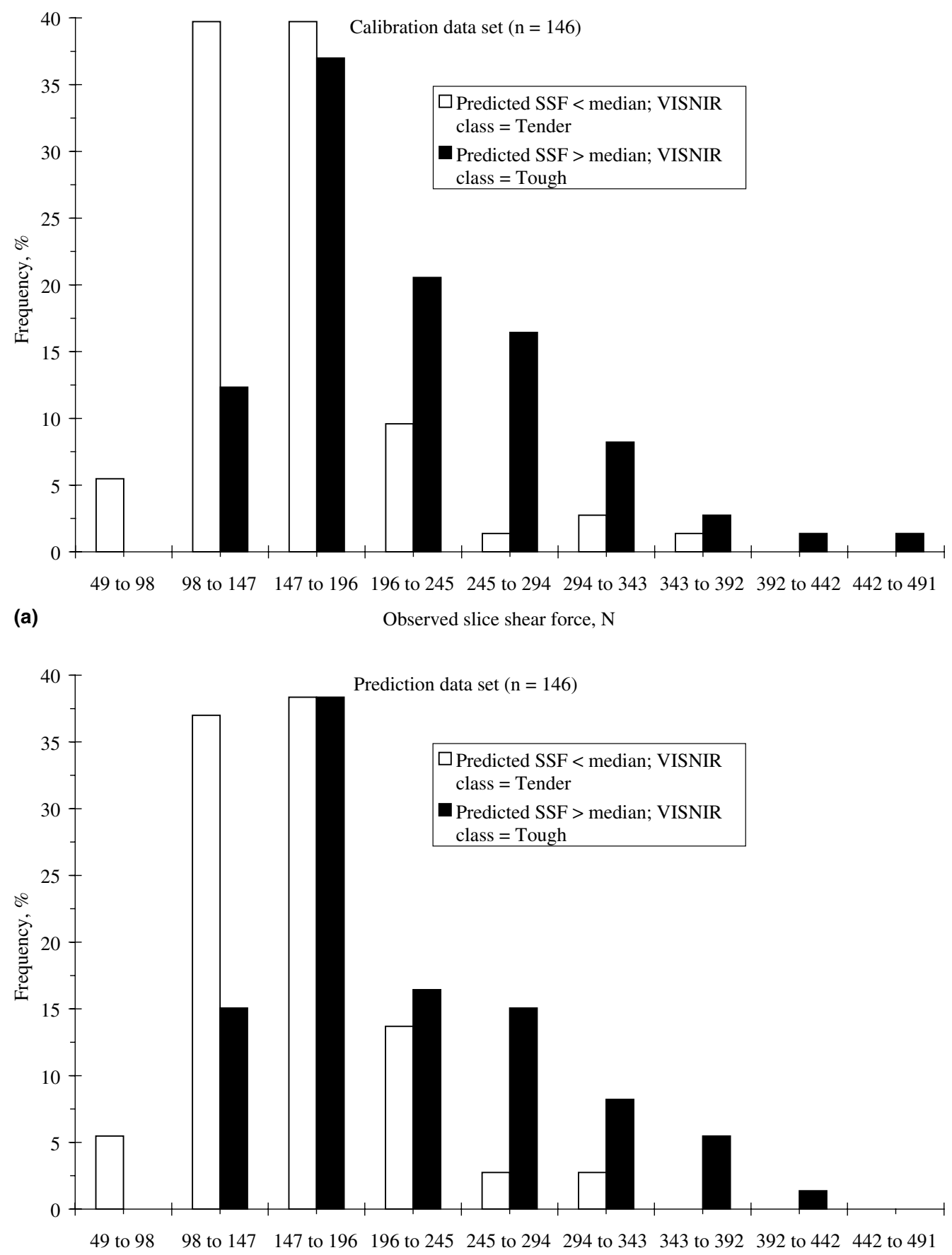

(b)

Observed slice shear force, $\mathrm{N}$

Fig. 2. Frequency distributions for observed slice shear force for the calibration (a) and prediction (b) data sets. 
percentage of carcasses with slice shear force values greater than $245 \mathrm{~N}$, higher trained sensory panel tenderness ratings, and a lower percentage of carcasses with trained sensory panel tenderness ratings below slightly tender (Table 2). Likewise, when the equation developed in the calibration data set was tested on the prediction data set, the tender class had a lower mean SSF value, a lower percentage of carcasses with slice shear force values greater than $245 \mathrm{~N}$, higher trained sensory panel tenderness ratings, and a lower percentage of carcasses with trained sensory panel tenderness ratings below slightly tender (Table 2). For the prediction data set, 22 of the $73(30.1 \%)$ carcasses with VISNIR-predicted SSF values greater than the median $(182 \mathrm{~N})$ had SSF values above $245 \mathrm{~N}$. In contrast, only 4 of the 73 $(5.5 \%)$ carcasses with VISNIR-predicted SSF values less than the median had SSF values above $245 \mathrm{~N}$. Likewise, whereas 26 of the $73(35.6 \%)$ carcasses with VISNIRpredicted SSF values greater than the median had sensory panel tenderness ratings below slightly tender, only 8 of the $73(11.0 \%)$ carcasses with VISNIR-predicted SSF values less than the median had sensory panel tenderness ratings below slightly tender. Further inspection of the frequency distributions of the slice shear force values of the VISNIR classes revealed that the frequency of carcasses in each $49 \mathrm{~N}$ increment from 196 to $491 \mathrm{~N}$ was numerically lower for the tender class (Fig. 2).

This technology was evaluated on US Select carcasses because (1) US Select cuts are currently marketed at a discount relative to US Choice cuts despite the fact that many cuts from US Select carcasses are very tender, (2) consumers have shown a strong willingness to pay a premium for "Tender Select" cuts that combine superior tenderness with the leanness of Select (Shackelford et al., 2001), (3) it is likely that higher degrees of marbling would interfere with the ability of VISNIR to predict tenderness and (4) identification of tender longissimus from US Select would increase the value of carcasses usually discounted because of assumed lower eating quality. To our knowledge, this is the first report of a system that is capable of non-invasively classifying US Select carcasses for longissimus tenderness with a useful level of accuracy. However, further testing is needed to determine whether the effectiveness of the VISNIR prediction achieved in the current experiment can be achieved in other populations.

\section{Conclusions}

The present experiment indicates that US Select carcasses can be non-invasively classified for longissimus tenderness using visible and near-infrared spectroscopy. This technology might be useful for identification of US Select carcasses that excel in longissimus tenderness for use in branded beef programs.

\section{Acknowledgments}

We are grateful to Patty Beska, Kathy Mihm, Pat Tammen, In-Ho Hwang, and Min-Suk Rhee for technical assistance and we are grateful to Marilyn Bierman and Carol Grummert for secretarial assistance.

\section{References}

Belk, K. E., Scanga, J. A., Wyle, A. M., Wulf, D. M., Tatum, J. D., \& Reagan, J. O., et al. (2000). The use of video image analysis and instrumentation to predict beef palatability. Proceeding of the Reciprocal Meat Conference, 53, 10-15.

Byrne, C. E., Downey, G., Troy, D. J., \& Buckley, D. J. (1998). Nondestructive prediction of selected quality attributes of beef by nearinfrared reflectance spectroscopy between 750 and $1098 \mathrm{~nm}$. Meat Science, 49, 399-409.

Cross, H. R., Moen, R., \& Stanfield, M. S. (1978). Training and testing of judges for sensory analysis of meat quality. Food Technology, 37, 48-54.

Hildrum, K. I., Nilsen, B. N., Mielnik, M., \& Naes, T. (1994). Prediction of sensory characteristics of beef by near-infrared spectroscopy. Meat Science, 38, 67-80.

Hildrum, K. I., Isaksson, T., Naes, T., Nilsen, B. N., Rodbotten, M., \& Lea, P. (1995). Near-infrared reflectance reflectance spectroscopy in the prediction of sensory propertiess of beef. Journal of Near Infrared Spectroscopy, 3, 81-87.

Mitsumoto, M., Maeda, S., Mitsuhashi, T., \& Ozawa, S. (1991). Nearinfrared spectroscopy determination of physical and chemical characteristics in beef cuts. Journal of Food Science, 56, 1493-1496.

Naes, T., \& Hildrum, K. I. (1997). Comparison of multivariate calibration and discriminant analysis in evaluating NIR spectroscopy for determination of meat tenderness. Applied Spectroscopy, 51, 350-357.

NAMP. (1997). The meat buyers guide (3rd ed.). Reston, VA: North American Meat Processors Association.

Neter, J., Wasserman, W., \& Kutner, M. H. (1989). Applied linear regression models (2nd ed.). Homewood, IL: Irwin.

Park, B., Chen, Y. R., Hruschka, W. R., Shackelford, S. D., \& Koohmaraie, M. (1998). Near-infrared reflectance analysis for predicting beef longissimus tenderness. Journal of Animal Science, 76, 2115-2120.

Savell, J. W., Cross, H. R., \& Smith, G. C. (1986). Percentage ether extractable fat and moisture content of beef longissimus muscle as related to USDA marbling score. Journal of Food Science, 51, $838-840$

Shackelford, S. D., Wheeler, T. L., \& Koohmaraie, M. (1999). Tenderness classification of beef. II: Design and analysis of a system to measure beef longissimus shear force under commercial processing conditions. Journal of Animal Science, 77, 1474-1481.

Shackelford, S. D., Wheeler, T. L., \& Koohmaraie, M. (2004). Development of optimal protocol for visible and near-infrared reflectance spectroscopic evaluation of meat quality. Meat Science, $68,371-381$.

Shackelford, S. D., Wheeler, T. L., Meade, M. K., Reagan, J. O., Byrnes, B. L., \& Koohmaraie, M. (2001). Consumer impressions of Tender Select beef. Journal of Animal Science, 79, 2605-2614.

Wheeler, T. L., Shackelford, S. D., \& Koohmaraie, M. (1998). Cooking and palatability traits of beef longissimus steaks cooked with a belt grill or an open hearth electric broiler. Journal of Animal Science, 76, 2805-2810.

Wheeler, T. L., Vote, D., Leheska, J. M., Shackelford, S. D., Belk, K. E., Wulf, D. M., et al. (2002). The efficacy of three objective 
systems for identifying beef cuts that can be guaranteed tender. Journal of Animal Science, 80, 3315-3327.

Wulf, D. M., \& Page, J. K. (2000). Using measurements of muscle color, $\mathrm{pH}$ and electrical impedance to augment the current USDA beef quality grading standards and improve the accuracy and precision of sorting carcasses into palatability groups. Journal of Animal Science, 78, 2595-2607. 